\title{
Three-dimensional Graphene-Carbon Nanotubes Composite- Supported PtSn Catalysts with a Tunable Microstructure to Enhance Electrocatalytic Activity for Ethanol Oxidation
}

\author{
Lili Wang ${ }^{1}$, Yanting Li $^{1}$, Xueli Miao ${ }^{1}$, Hao Qin ${ }^{1}$, Jiahao Ren ${ }^{1}$, Xiaofeng Wang ${ }^{2}$, Zhichun Sun ${ }^{3}$, \\ Bing Wang ${ }^{1, *}$ \\ ${ }^{1}$ State Key Laboratory of Separation Membranes and Membrane Processes, School of Environment \\ and Chemical Engineering, Tianjin Polytechnic University, 399 Binshui West Road, Tianjin 300387, P. \\ R. China \\ ${ }^{2}$ State Key Laboratory of Inorganic Synthesis and Preparative Chemistry, College of Chemistry, Jilin \\ University, Changchun 130012, P. R. China \\ ${ }^{3}$ Sinosteel Tianjin Geological Research Institute Limited Company, Tianjin 300181, China \\ *E-mail: bingwang@tipu.edu.cn
}

doi: $10.20964 / 2018.11 .98$

Received: 10 August 2018 / Accepted: 11 September 2018 / Published: 1 October 2018

In this paper, nanostructured platinum-stannum ( $\mathrm{PtSn})$ dispersed on a series of composites of grahene oxide (GO) and multi-walled carbon nanotubes (MWNTs), denoted as PtSn/G-CNT have been prepared. Tunable size and distribution of the PtSn nanoparticles was achieved by adjusting the ratio of GO and MWNTs in the composites. The synthesized PtSn/G-CNT, single graphene (PtSn/G) and MWNTs (PtSn/CNT) were applied as anode catalysts for ethanol oxidation. The surface morphology and microstructure of as-prepared catalysts were investigated by scanning electron microscopy, transmission electron microscopy, X-ray photoelectron spectroscopy and X-ray diffraction. The electrocatalytic performance for ethanol oxidation has been estimated by cyclic voltammetry and chronoamperometry in acid media. The PtSn/G-CNT catalysts exhibit higher electrocatalytic activity and stability compared to the PtSn/G and PtSn/CNT. Among all the PtSn/G-CNT catalysts, the optimal $\mathrm{PtSn} /(2 / 3 \mathrm{G}+1 / 3 \mathrm{CNT})$ catalyst with narrower size, more uniform distribution and higher content of PtSn nanoparticles exhibited higher electrocatalytic activity and stability, which implied the application prospect in the field of ethanol fuel cells.

Keywords: Graphene oxide; Carbon nanotubes; PtSn nanoparticles; Composite support; Electrocatalytic performance

\section{FULL TEXT}


(C) 2018 The Authors. Published by ESG (www.electrochemsci.org). This article is an open access article distributed under the terms and conditions of the Creative Commons Attribution license (http://creativecommons.org/licenses/by/4.0/). 